\title{
Ultrasound-Responsive Polymer-Coated Microbubbles That Bind and Protect DNA
}

\author{
Ine Lentacker, Bruno G. De Geest, Roosmarijn E. Vandenbroucke, Liesbeth Peeters, \\ Joseph Demeester, Stefaan C. De Smedt,* and Niek N. Sanders \\ Laboratory of General Biochemistry and Physical Pharmacy, Faculty of Pharmacy, Ghent University, \\ Harelbekestraat 72, B-9000 Ghent, Belgium
}

Received February 9, 2006. In Final Form: June 7, 2006

\begin{abstract}
Ultrasound in combination with microbubbles has recently been considered by gene delivery scientists to be an interesting approach to enhance gene transfer into cells. Its low toxicity and simplicity to apply in vivo without major complications make this technology (sonoporation) especially attractive. Sonoporation of DNA has been evaluated in vivo by the injection of free plasmid DNA (pDNA) together with microbubbles (as used in diagnostic imaging) in the bloodstream. However, the in vivo gene-transfer efficiency in these experiments remained rather low. Both the enzymatic degradation of the injected pDNA as well as the low pDNA concentration in the neighborhood of sonoporated cell membranes may explain this low efficiency. Therefore, we developed polymer-coated microbubbles that can bind and protect the pDNA. Coating albumin-shelled microbubbles with poly(allylamine hydrochloride) (PAH) makes the surface charge of the microbubbles positive without drastically affecting the size distribution of the microbubbles, thereby not affecting the ultrasound responsiveness and injectability. The cationic coating allowed both to bind up to $0.1 \mathrm{pg}$ of DNA per microbubble as well as to protect the bound DNA against nucleases. Finally, the PAH coating significantly increased the lifetime of the microbubbles (half-life $\approx 7 \mathrm{~h}$ ), making them more convenient for in vivo applications because more microbubbles are expected to reach the target organ. Binding and nuclease protection of DNA by polymer-coated diagnostic microbubbles has, to our knowledge, never been demonstrated. We conclude that these LbL-coated microbubbles might be significant in the further development of ultrasound-mediated gene delivery.
\end{abstract}

\section{Introduction}

Gene therapy was put forward in the late 1980s as the most promising therapy for genetic diseases. However, gene therapy is currently caught in a bottleneck because of the lack of efficient and safe gene carriers. DNA molecules are large, negatively charged molecules and have major difficulties in entering the cell or cell nucleus. On top, DNA becomes rapidly degraded by extra- and intracellular nucleases. ${ }^{1}$ Therefore, suitable DNA delivery systems are under development. The first system makes use of replication-deficient viruses that accommodate the therapeutic DNA in their genome. ${ }^{1}$ These viral gene carriers transfect very efficiently because they have an ingenious system for the nuclear delivery of exogenous DNA. However, viral gene carriers have some important disadvantages: they often provoke an immune response and severe inflammation reactions. ${ }^{2}$ Additionally, the risk for insertional mutagenesis and the size limitation of the DNA that they can accommodate are other drawbacks of viral gene carriers. ${ }^{1}$ Therefore, nonviral transfection systems based on cationic lipids or cationic polymers have gained more and more attention. ${ }^{1}$ Although nonviral carriers may be safer and cheaper, they have, especially in vivo, a much lower transfection efficiency than viral gene carriers.

As outlined above, the in vivo application of viral and nonviral DNA delivery systems is currently hampered by safety concerns and low efficiency, respectively. To overcome the limitations of nonviral gene therapy, ultrasound energy, alone or in combination with gas-filled microbubbles, has recently been proposed to enhance the intracellular delivery of DNA, siRNA, and proteins. $^{3-12}$ Because ultrasound energy in combination with gas-filled microbubbles has been used for several years in medical

* Corresponding author. E-mail: stefaan.desmedt@ugent.be. Tel: +32(0)9/2648047. Fax: +32(0)9/2648189.

(1) Patil, S. D.; Rhodes, D. G.; Burgess, D. J. AAPS J. 2005, 7, E61

(2) Marshall, E. Science 1999, 286, 2244. imaging, it can be considered to be very safe. ${ }^{13}$ The mechanism by which ultrasound mediates intracellular drug delivery has been ascribed as cavitation, which is the alternate growing and shrinking of gas-filled microbubbles as a result of the high- and low-pressure waves generated by ultrasound energy. Finally, these "cavitating" (oscillating) microbubbles implode. The cavitation and especially the implosion of the microbubbles generate local shock waves and microjets that can temporarily perforate the cell membrane, allowing macromolecules to enter the cells. ${ }^{14-18}$ This ultrasound-assisted delivery of macromolecules, often called sonoporation, has been proved to be effective both in vitro and in vivo. ${ }^{3-12}$ However, a major limitation of the currently available microbubbles is that they have a short lifetime

(3) Bekeredjian, R.; Chen, S. Y.; Grayburn, P. A.; Shohet, R. V. Ultrasound Med. Biol. 2005, 31, 687.

(4) Chen, S. Y.; Shohet, R. V.; Bekeredjian, R.; Frenkel, P.; Grayburn, P. A J. Am. Coll. Cardiol. 2003, 42, 301.

(5) Duvshani-Eshet, M.; Machluf, M. J. Controlled Release 2005, 108, 513. 393.

(7) Kinoshita, M.; Hynynen, K. Pharm. Res. 2005, 22, 716.

(8) Manome, Y.; Nakayama, N.; Nakayama, K.; Furuhata, H. Ultrasound Med. Biol. 2005, 31, 693.

(9) Mehier-Humbert, S.; Bettinger, T.; Yan, F.; Guy, R. H. J. Controlled Release 2005, 104, 203.

(10) Newman, C. M.; Lawrie, A.; Brisken, A. F.; Cumberland, D. C. Echocardiogr.-J. Card. 2001, 18, 339.

(11) Pislaru, S. V.; Pislaru, C.; Kinnick, R. R.; Singh, R.; Gulati, R.; Greenleaf, J. F.; Simari, R. D. Eur. Heart J. 2003, 24, 1690.

(12) Vannan, M.; McCreery, T.; Li, P.; Han, Z. G.; Unger, E.; Kuersten, B.; Nabel, E.; Rajagopalan, S. J. Am. Soc. Echocardiogr. 2002, 15, 214.

(13) Grayburn, P. A. Echocardiogr.-J. Card. 2002, 19, 259.

(14) Christiansen, J. P.; French, B. A.; Klibanov, A. L.; Kaul, S.; Lindner, J.

R. Ultrasound Med. Biol. 2003, 29, 1759.

(15) Marmottant, P.; Hilgenfeldt, S. Nature 2003, 423, 153.

(16) Mehier-Humbert, S.; Bettinger, T.; Yan, F.; Guy, R. H. J. Controlled Release 2005, 104, 213.

(17) Tachibana, K.; Uchida, T.; Ogawa, K.; Yamashita, N.; Tamura, K. Lancet 1999, 353, 1409.

(18) Van Wamel, A.; Bouakaz, A.; Versluis, M.; De Jong, N. Ultrasound Med. Biol. 2004, 30, 1255 . 
and neither bind nor protect the therapeutic DNA against nucleases. Binding of the DNA on the microbubbles will ensure that the DNA is present at the site of cell membrane poration, enhancing the chance that the DNA is dragged inside the cell or even inside the nucleus by the generated microjets.

The aim of this work is to develop ultrasound-responsive microbubbles that (a) can bind the DNA, (b) protect the DNA against nucleases, and (c) remain stable for several hours. Therefore, we coated, to our knowledge for the first time, perfluorcarbon gas-filled microbubbles with a cationic polymer via the layer-by-layer (LbL) technique. ${ }^{19} \mathrm{We}$ characterized the physical properties, ultrasound responsiveness, DNA binding, and DNA protection toward nucleases of this new type of microbubble.

\section{Materials and Methods}

2.1. Preparation of Microbubbles and PAH-Coated Microbubbles. Microbubbles were prepared following the procedure developed by Porter et al. ${ }^{20}$ Briefly, one part of a $5 \%$ bovine serum albumin (Sigma Aldrich, St Louis, MO) solution in HEPES buffer ( $20 \mathrm{mM}, \mathrm{pH} 7.4$ ) was mixed with two parts of a 5\% dextrose (Sigma Aldrich, St Louis, MO) solution in HEPES buffer. Subsequently, the mixture was drawn into a $30 \mathrm{~mL}$ syringe and blended with 10 $\mathrm{mL}$ of perfluorobutane (MW $238 \mathrm{~g} / \mathrm{mol}$, F2 chemicals, Preston, Lancashire, U.K.) through a three-way valve. After mixing by hand, the solution was sonicated with a $20 \mathrm{kHz}$ probe (Branson 250 sonifier, Branson Ultrasonics Corp., Danbury, CT). Following sonication, the microbubbles were centrifuged at $118 g$ for $1 \mathrm{~min}$. The subnatants were discarded, and the microbubbles were washed three times with sterile HEPES buffer. Finally, the microbubbles were suspended in $5 \mathrm{~mL}$ of sterile HEPES buffer.

The PAH-coated microbubbles were prepared by the layer-bylayer (LbL) coating of the microbubbles obtained above. Five milliliters of a microbubble dispersion was incubated with $5 \mathrm{~mL}$ of a poly(allylamine hydrochloride) (PAH, MW 70000 g/mol, SigmaAldrich) solution ( $2 \mathrm{mg} / \mathrm{mL}$, HEPES buffer). Subsequently, the PAH was removed by washing (three times) the microbubbles with sterile HEPES buffer. Therefore, after each wash step the microbubbles were centrifuged at $118 \mathrm{~g}$ for $1 \mathrm{~min}$. Finally, the LbL-coated microbubbles were suspended in $5 \mathrm{~mL}$ of sterile HEPES buffer.

2.2. Characterization of the Microbubbles. The concentration of the microbubble dispersions was determined immediately after their preparation with the aid of a Burker chamber and a light microscope. The concentrations of the uncoated and coated microbubble dispersions were $9.57 \pm 0.65 \times 10^{8}$ and $1.99 \pm 0.10 \times$ $10^{8}$ microbubbles $/ \mathrm{mL}$, respectively. To visualize the microbubbles, they were brought into a $\mu$-slide VI flow chamber (Ibidi Integrated BioDagnostics, München, Germany) and studied via light (Nikon TS100-F, Melville, NY) or confocal laser scanning microscopy (CLSM) using a $40 \times$ lens. The size distribution of the microbubbles was determined within 10 min after preparation by laser diffraction (Mastersizer S, Malvern, Worcestershire, U.K.). The zeta potential of the microbubbles was measured by particle electrophoresis (Zetasizer 2000, Malvern, Worcestershire, U.K.). All the experiments were performed on microbubbles dispersed in HEPES buffer (20 $\mathrm{mM}, \mathrm{pH}$ 7.4).

2.3. Plasmid DNA Preparation. The plasmid DNA (pDNA; pGL3, Promega, Leiden, The Netherlands) used in this study contained as a reporter gene luciferase from Photinus pyralis under the control of a simian virus 40 promotor. After amplification of the pDNA in Escherichia coli, the pDNA was extracted and purified from the bacterial cells using the Qiagen giga kit (Valencia, CA). The pDNA concentration was set at $1.0 \mathrm{mg} / \mathrm{mL}$ HEPES buffer assuming that the absorption at $260 \mathrm{~nm}$ of a $50 \mu \mathrm{g} / \mathrm{mL}$ DNA solution equals 1 . The pDNA showed a high purity because the ratio of the absorptions at 260 and $280 \mathrm{~nm}$ was between 1.8 and 2.0.

(19) Decher, G. Science 1997, 277, 1232. 577 .

(20) Porter, T. R.; Iversen, P. L.; Li, S.; Xie, F. J. Ultrasound Med. 1996, 15 ,
2.4. Fluorescent Labeling of Albumin, PAH, and pDNA. Bovine serum albumin (BSA, Sigma-Aldrich) was labeled with fluoresceine isothiocyanate (FITC; Sigma-Aldrich) by vigorous mixing of 60 $\mathrm{mL}$ of FITC solution $(0.2 \mathrm{mg} / \mathrm{mL} 0.1 \mathrm{M}$ borate buffer at $\mathrm{pH} 8.5)$ with $60 \mathrm{~mL}$ of BSA $(5 \mathrm{mg} / \mathrm{mL} 0.1 \mathrm{M}$ borate buffer at $\mathrm{pH} 8.5)$. The labeling of PAH with rhodamine isothiocyanate (RITC) occurred in a similar way. Twelve milligrams of RITC and $300 \mathrm{mg}$ of PAH were separately dissolved in $60 \mathrm{~mL}$ of borate buffer $(0.1 \mathrm{M}, \mathrm{pH} 8.5)$ and subsequently mixed under vigorous stirring. After overnight incubation, the reaction mixtures were dialyzed (MW cutoff of the membrane was $25 \mathrm{kDa}$ ) against pure water for several days. Finally, the dialyzed FITC-BSA and RITC-PAH were freeze dried, and the resulting fluffy solids were stored at $4{ }^{\circ} \mathrm{C}$.

Intercalating dyes YOYO-1 and TOTO- 3 were used to label the pDNA. Therefore, $111 \mu \mathrm{g}$ of pDNA was mixed with $57 \mu \mathrm{L}$ of a 1/100 diluted YOYO-1 or TOTO-3 solution (Molecular Probes, Eugene, OR) and diluted in TE buffer $(10 \mathrm{mM}$ tris- $\mathrm{HCl} ; 1 \mathrm{mM}$ EDTA; $\mathrm{pH}$ 7.4) until a final pDNA concentration of $1 \mathrm{mg} / \mathrm{mL}$ was obtained. The dye/base pair ratio was 1:30 for both the YOYO-1 and TOTO-3 labeled pDNA. For the FCS measurements, the pDNA was labeled with cy-5, using the Mirus labeling kit (Mirus Bio Corporation, Madison, WI). The dye/base pair ratio was 1:2.

2.5. Confocal Laser Scanning Microscopy. The microbubbles, put in $\mu$-slide VI-flow chambers, were visualized by a confocal laser scanning microscope (CLSM, BioRad MRC 1024, Hemel Hempstadt, U.K.) equipped with a krypton-argon laser and a dichroic mirror that reflects the laser light in a $40 \times$ objective. The $488 \mathrm{~nm}$ line of this laser was used to excite YOYO-1 and FITC, and the $568 \mathrm{~nm}$ line was used to excite RITC. To ensure a proper spectral separation, appropriate emission filters were used before the green and red detector.

2.6. Electron Microscopy. Five microliters of a coated microbubble dispersion was applied on a silicon wafer and air dried. The remains of the microbubbles were then examined with a scanning electron microscope (Quanta 200 FEG, FEI Company, Hillsboro, Oregon).

2.7. PicoGreen Assay and Fluorescence Fluctuation Spectroscopy. To determine the maximal pDNA loading capacity of the microbubbles, we mixed $150 \mu \mathrm{L}$ of the uncoated or coated microbubble dispersions with increasing amounts of pDNA. After 10 min of incubation, the microbubbles (with pDNA) were centrifuged at $118 \mathrm{~g}$, and the concentration of unbound pDNA in the subnatants was determined using PicoGreen (Invitrogen, Merelbeke, Belgium) and fluorescence correlation spectroscopy (FCS).

For the PicoGreen assay, $50 \mu \mathrm{L}$ of the subnatants was incubated with $1 \mathrm{~mL}$ of diluted (200-fold in TE buffer) PicoGreen reagent for $5 \mathrm{~min}$, and subsequently the fluorescence was determined $\left(\lambda_{\text {excitation }}=\right.$ $480 \mathrm{~nm}$ and $\lambda_{\text {emission }}=520 \mathrm{~nm}$ ).

We also determined the maximal pDNA loading capacity in another way, making use of fluorescence fluctuation spectroscopy (FFS) to measure the fluorescence of the unbound pDNA in the subnatants. FFS monitors the fluorescence fluctuations in the excitation volume of the microscope. The fluorescence signal is fluctuating because of the diffusion of fluorescent molecules in and out of the excitation volume. From the fluorescence fluctuations, an autocorrelation curve can be derived that allows one to calculate both the diffusion coefficient of the fluorescent molecules as well as the number of fluorescent molecules in the excitation volume. ${ }^{21}$ Details of the FFS setup used are explained in our earlier reports. ${ }^{22}$ For these experiments, Cy5-labeled pDNA was used, and samples were excited with $6 \%$ laser intensity $(647 \mathrm{~nm})$. First, a diluted solution of Cy5-pDNA was measured. Afterwards, $50 \mu \mathrm{L}$ of a coated bubble suspension was incubated with $2 \mu \mathrm{g}$ of pDNA. After $5 \mathrm{~min}$ of incubation, the sample was diluted to the same Cy5-pDNA concentration, and the amount of free pDNA was determined via FCS.

2.8. Ultrasound Responsiveness of the Microbubbles. The ultrasound responsiveness of pDNA-loaded, PAH-coated mi-

(21) Schwille, P.; Haustein, E. Biophysics Textbook, online 2006.

(22) Lucas, B.; Van Rompaey, E.; De Smedt, S. C.; Demeester, J.; Van Oostveldt, P. Macromolecules 2002, 35, 8152. 


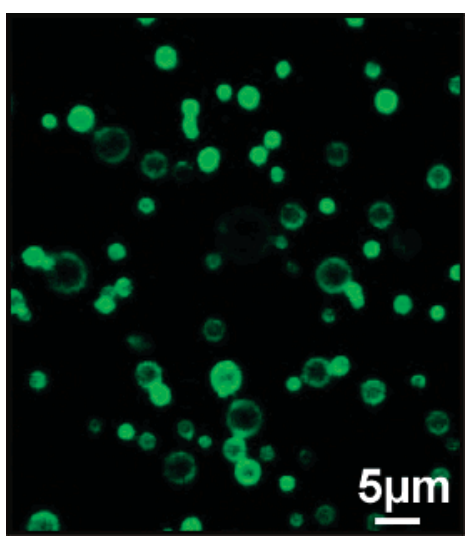

Figure 1. Confocal fluorescence microscopy image of perfluorocarbon microbubbles stabilized with FITC-labeled albumin. Depending on the position of the confocal plane in the microbubbles, we observed fluorescent rings (confocal plane in the middle of the microbubbles) or filled circles (confocal plane at the top or bottom of the microbubbles).

crobubbles was determined by comparing their (number) concentration before and after exposure to ultrasound. pDNA-loaded microbubbles were prepared by mixing $100 \mu \mathrm{L}$ of a PAH-coated microbubble dispersion with $2 \mu \mathrm{g}$ of pDNA. After $10 \mathrm{~min}$ of incubation, the concentration of the pDNA-loaded microbubbles was determined in a Burker chamber. Subsequently, the pDNAloaded microbubbles were sonicated $\left(1 \mathrm{MHz}, 1 \mathrm{~W} / \mathrm{cm}^{2} ; 50 \%\right.$ duty cycle) for $30 \mathrm{~s}$ using a Sonitron 2000 (RichMar, Inola, Oklahoma) and again counted in a Burker chamber.

2.9. Gel Electrophoresis to Evaluate the Stability of Microbubble-Bound pDNA to rhDNase I. To determine whether the microbubbles were able to protect the pDNA against rhDNase I (Pulmozyme, Roche, Belgium), gel electrophoresis experiments were performed. Two micrograms of pDNA was mixed with $50 \mu \mathrm{L}$ of a PAH-coated microbubble dispersion. After 5 min of incubation, the pDNA/microbubble mixtures were diluted in HEPES buffer supplied with $110 \mathrm{mM}$ potassium acetate and $2 \mathrm{mM}$ magnesium acetate ( $\mathrm{pH} 7.4)$, which is necessary to activate the rhDNase I. Subsequently, rhDNase I was incubated with the pDNA/microbubbles mixtures for $15 \mathrm{~min}$ at room temperature. The rhDNase I activity in the microbubble dispersions was $200 \mathrm{U} / \mathrm{L}$. Microbubble dispersions "incubated with inhibited rhDNase I" received $8 \mu \mathrm{L}$ of $\mathrm{Na}_{2}$ EDTA (50 $\mathrm{mM}$ ) before the addition of the rhDNase I to the pDNA/ microbubble mixtures. $\mathrm{Na}_{2}$ EDTA inhibits rhDNase I by complexing the divalent cations, which are required for activating rhDNase I. Other samples were supplemented with $\mathrm{Na}_{2}$ EDTA after incubation with rhDNase I.

After the incubation period with rhDNase I, the pDNA was released from the microbubbles to investigate whether it survived the exposure to rhDNase I. Therefore, $\mathrm{NaCl}$ was added to the $\mathrm{pDNA} /$ microbubbles dispersions at a final concentration of $5 \mathrm{M}$. Subsequently, the pDNA/ microbubbles dispersions were centrifuged for $1 \mathrm{~min}$ at $118 \mathrm{~g}$. Fifty microliters of the subnatants was mixed with $10 \mu \mathrm{L}$ of a $30 \%$ glycerol solution and loaded onto a $1.5 \%$ agarose gel prepared in TBE (10.8 $\mathrm{g} / \mathrm{L}$ tris base, $5.5 \mathrm{~g} / \mathrm{L}$ boric acid, and $0.58 \mathrm{~g} / \mathrm{L}$ EDTA). The samples were subjected to electrophoresis at $100 \mathrm{~V}$ for $60-90 \mathrm{~min}$, and the pDNA was visualized by ethidium bromide $(0.5 \mu \mathrm{g} / \mathrm{mL})$ staining prior to UV photography.

\section{Results and Discussion}

3.1. Preparation and Characterization of Uncoated and PAH-Coated Microbubbles. After the preparation of perfluorocarbon microbubbles stabilized with (FITC-labeled) albumin, CLSM experiments revealed the existence of micrometer-sized spherical structures covered with green-labeled albumin (Figure 1) that floated atop the liquid. Because the size of the microbubbles determines their ability to serve as cavitation nuclei, we subs-

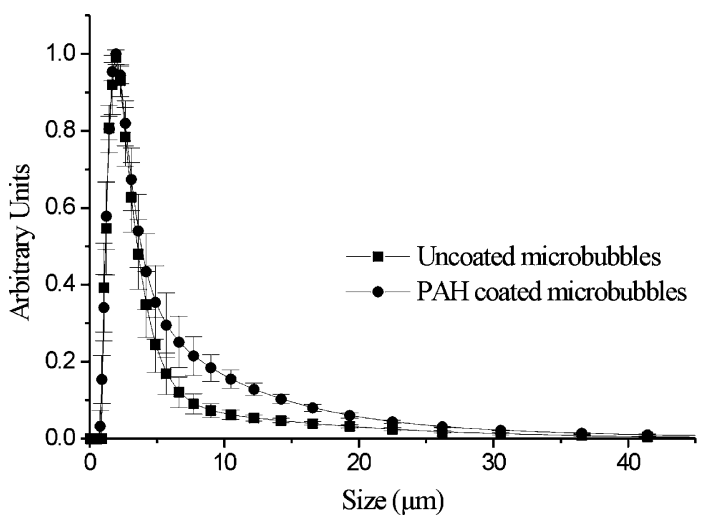

Figure 2. Size distribution of uncoated and PAH-coated albumin/ perfluorocarbon microbubbles as measured by laser diffraction. The $y$ axis shows to what extent a certain class of microbubbles is present, normalized to the most abundant fraction of microbubbles $(y=1)$. Data are the means of three independent measurements.

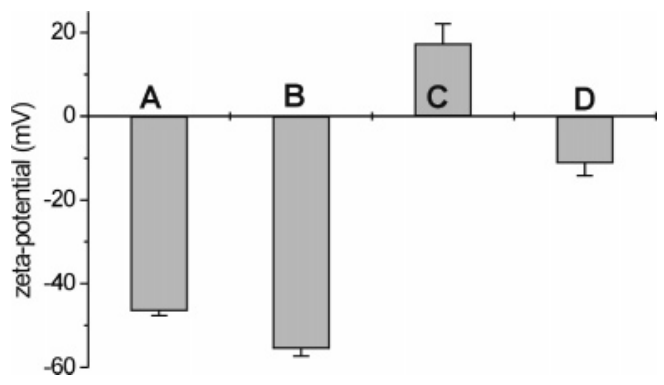

Figure 3. Zeta potential of uncoated microbubbles (A), uncoated microbubbles $(50 \mu \mathrm{L})$ incubated with $1 \mu \mathrm{g}$ of pDNA (B), PAHcoated microbubbles $(\mathrm{C})$, and PAH-coated bubbles $(50 \mu \mathrm{L})$ incubated with $1 \mu \mathrm{g}$ of pDNA (D). The data are the means of three independent measurements.

equently studied the size distribution of freshly made microbubbles by laser diffraction. ${ }^{23}$ Most of the albumin-stabilized microbubbles (about 90\%) were between 1 and $5 \mu \mathrm{m}$ in diameter (Figure 2), which is a size known to favor cavitation upon exposure to clinically used ultrasound frequencies (such as $1 \mathrm{MHz}$ ). ${ }^{23}$

The albumin chains, which cover the gaseous cores, stabilize the microbubbles because they reduce the diffusion of the perfluorocarbon gas out of the microbubbles in the surrounding water. The shell-forming properties of albumin most likely originate from its amphiphilic nature. Indeed, one can expect that the hydrophobic regions of albumin have an affinity for the perfluorocarbon gas, whereas its negatively charged hydrophilic parts protrude into the aqueous phase. The presence of negative charges at the surface of the albumin-stabilized microbubbles was indeed confirmed from zeta potential $(\zeta)$ measurements: the average $\zeta$ equalled $-45 \mathrm{mV}$ (Figure 3 ).

One can expect that the negative charges at the microbubbles' surface would enable them to be coated with cationic polymers. Therefore, we tried to apply a positively charged layer around the albumin shell using PAH, a polycation that is widely used in the LbL coating of planar substrates and colloidal templates. $^{24-26}$ Figure 4 shows CLSM images of microbubbles prepared with (nonfluorescently labeled) albumin and coated with RITC-labeled PAH. Clearly, the PAH chains cover the outer surface of the microbubbles and are not incorporated into the perfluorocarbon gas core. Also, after the PAH coating, the zeta potential of the microbubbles became positive $(15 \mathrm{mV}$; Figure 3), which further confirms the presence of $\mathrm{PAH}$ at the

(23) Unger, E. C.; Porter, T.; Culp, W.; Labell, R.; Matsunaga, T.; Zutshi, R. Adv. Drug Delivery Rev. 2004, 56, 1291. 


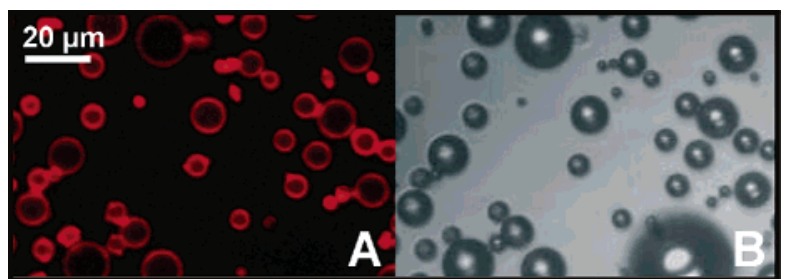

Figure 4. Confocal fluorescence microscopy image (A) and transmission image (B) of PAH-coated microbubbles made with unlabeled albumin and RITC-labeled PAH.

surface of the microbubbles. Apart from a slightly elevated fraction in the 5-20 $\mu \mathrm{m}$ range, which is probably due to the aggregation of smaller microbubbles after coating, the $\mathrm{PAH}$ coating changed the size distribution of the microbubbles only moderately (Figure 2). In the remainder of this article, we use the terms "uncoated: and "PAH-coated" microbubbles to refer to albumin/perfluorocarbon microbubbles without and with a PAH coating, respectively.

3.2. Stability of Uncoated and PAH-Coated Microbubbles. It is well known that the lifetime of air-filled albumin-stabilized microbubbles is very short because the air diffuses rapidly out of the microbubbles. ${ }^{27}$ The use of perfluorocarbon gas, which has a lower water solubility than air, effectively delays gas diffusion. ${ }^{28}$ Nevertheless, their lifetime remains very short. Coating microbubbles with PAH should offer a solution to this problem because polyelectrolyte multilayers have been reported to prevent or drastically decrease gas diffusion. ${ }^{29}$

Therefore, we evaluated the stability of the microbubbles at room temperature by following the concentration of the microbubbles as a function of time. Figure 5A shows the percentage of remaining uncoated and PAH-coated microbubbles as a function of time. Uncoated microbubbles seem to destabilize very rapidly: after $75 \mathrm{~min}, 50 \%$ of the uncoated microbubbles have already disappeared. In contrast, PAH-coated microbubbles clearly existed for much longer times: half of the PAH-coated microbubbles had disappeared after $6 \mathrm{~h}$.

3.3. DNA Binding Properties of the Microbubbles. The pDNA binding properties of the microbubbles were first evaluated by CSLM. To enable the visualization of the binding of pDNA to the microbubbles, YOYO-1-labeled pDNA (green) and RITClabeled PAH (red) were used. Uncoated and PAH-coated albumin microbubbles were incubated with pDNA for $2 \mathrm{~min}$, transferred to a $\mu$-slide VI flow chamber, and studied via CLSM. Figure 6

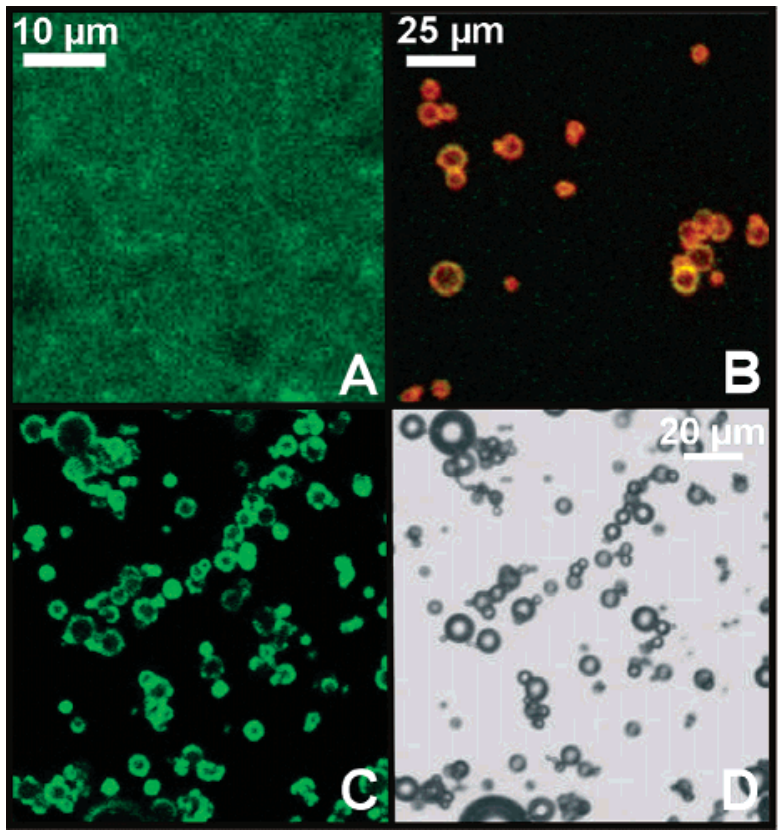

Figure 6. (A) Confocal fluorescence images of uncoated microbubbles $(50 \mu \mathrm{L})$ incubated with $1 \mu \mathrm{g}$ of YOYO-1-labeled pDNA, (B) RITC-labeled PAH-coated microbubbles $(50 \mu \mathrm{L})$ incubated with $2.6 \mu \mathrm{g}$ of YOYO-1-labeled pDNA, and (C) unlabeled PAH-coated microbubbles $(50 \mu \mathrm{L})$ incubated with $1 \mu \mathrm{g}$ of YOYO-1-labeled pDNA. (D) Transmission image corresponding to image C.

shows the results. Clearly, the green-labeled pDNA does not bind to the uncoated albumin microbubbles but remains in solution (Figure 6A). However, the green-labeled pDNA does bind to the RITC-PAH-coated microbubbles (Figure 6B) because yellow rings are present around the microbubbles, indicating the co-
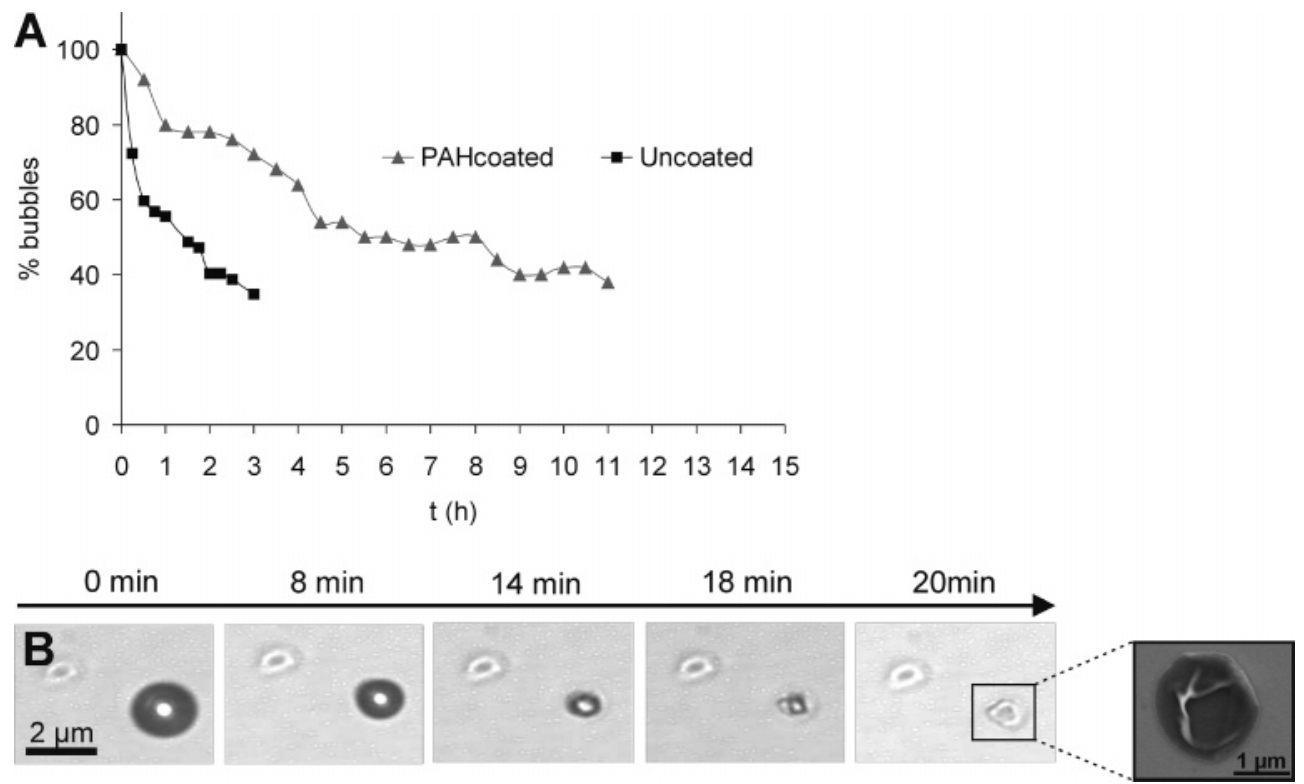

Figure 5. (A) Stability of uncoated and PAH-coated microbubbles. The concentration of the microbubbles was measured as a function of time and normalized to the concentration just after preparation. The dispersions were placed at room temperature under continuous stirring at $750 \mathrm{rpm}$, and the concentration of microbubbles was determined microscopically using a Burker chamber. (B) Light microscopy images of a destabilizing PAH-coated microbubble. The inset is an SEM image of the remaining polymer coating. Gradual shrinking was also observed on uncoated microbubbles, although it occurred faster. 
localization of pDNA and PAH. Between the microbubbles in Figure $6 \mathrm{~B}$, some green fluorescence (i.e., unbound pDNA) is still detected. This could be expected because the maximal pDNA loading capacity of the microbubbles was exceeded (see below). When the microbubbles (in this experiment, unlabeled) were incubated with lower amounts of YOYO-1-labeled pDNA, green fluorescence between the microbubbles was no longer observed (Figure 6C).

Successful loading of the PAH-coated microbubbles with pDNA could also be observed from zeta potential measurements because the addition of pDNA turned the zeta potential of the PAH-coated microbubbles negative (Figure 3 ). This most likely indicates that pDNA is bound to the surface of the PAH-coated microbubbles. In contrast, the addition of pDNA to the uncoated albumin microbubbles did not drastically alter the zeta potential of the uncoated microbubbles (Figure 3), which is in line with the observations above. Previously, Porter et al. reported that oligonucleotides do bind to albumin microbubbles. However, these authors used phosphorothioate-modified oligonucleotides, which are known to interact nonspecifically with proteins. ${ }^{30}$

To estimate the maximal pDNA binding capacity of the PAHcoated microbubbles, they were incubated (for $5 \mathrm{~min}$ ) with increasing amounts of pDNA, and the amount of unbound (i.e., free) pDNA in the subnatants (obtained after centrifugation) was determined via both the PicoGreen assay and FCS. Both tests revealed that $50 \mu \mathrm{L}$ of the $\mathrm{PAH}$-coated microbubble dispersion could maximally bind $1 \mu \mathrm{g}$ of pDNA (data not shown). Taking into account that $50 \mu \mathrm{L}$ of microbubble dispersion contains about $9.95 \times 10^{6}$ microbubbles, one can estimate that the PAH coating of the microbubbles enables them to carry $0.1 \mathrm{pg}$ of pDNA or about 20000 pDNA molecules per microbubble. These experiments prove that coating the microbubbles with PAH enables a very efficient loading of albumin microbubbles with plasmid DNA. As mentioned above, pDNA-loaded microbubbles have also been developed by other groups. Christiansen et al. and Vannan et al. prepared cationic microbubbles using lipids as a shell-forming material. ${ }^{14,12}$ Compared to PAH-coated microbubbles, these microbubbles have a 2.5 -fold lower pDNA loading capacity. However, we should take into account that in their studies the average microbubble size was smaller, which automatically implies a lower DNA loading. Also, cationic lipidbased microbubbles are probably more expensive. Frenkel et al. showed that the sonication of a solution of dextrose, albumin, and pDNA with perfluoropropane gas leads to microbubbles that contain albumin and pDNA in their shell. ${ }^{31}$ However, a major drawback of this technique is that only a small fraction of the pDNA seems to become incorporated into the shell.

3.4. Ultrasound Responsiveness and DNase Protection. Microbubbles enhance the efficiency of ultrasound-assisted gene delivery as they generate microjets that, upon implosion, temporarily perforate the cell membranes. ${ }^{14-18}$ Given the importance of cavitation, the ultrasound responsiveness of the uncoated and pDNA-loaded PAH-coated microbubbles was studied by exposing them for $30 \mathrm{~s}$ to ultrasound energy $(1 \mathrm{MHz}$, $1 \mathrm{~W} / \mathrm{cm}^{2}, 50 \%$ duty cycle). After ultrasound radiation, 99 and $95 \%$ of the uncoated and PAH-coated microbubbles, respectively, were destroyed, which is in agreement with previous reports. ${ }^{9,20}$ Despite their higher stability (Figure 5), PAH-coated microbubbles are thus clearly ultrasound-responsive.

Subsequently, the ability of the microbubbles to protect pDNA against nucleases was tested using gel electrophoresis. As described in the Materials and Methods section, the microbubble dispersions were exposed to $200 \mathrm{U} / \mathrm{L}$ rhDNase-I, which is about 20 -fold higher than the DNase activity found in human blood. ${ }^{32}$
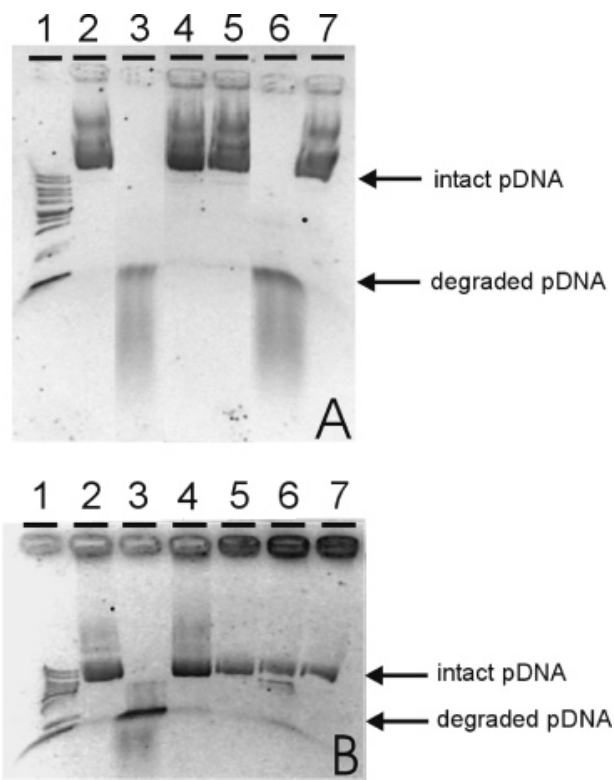

Figure 7. Gel electrophoresis of pDNA present in an uncoated and PAH-coated microbubble dispersion with or without DNase-I, respectively. The data obtained with uncoated microbubbles are shown by gel A, and those with PAH-coated microbubbles, by gel B. Each lane contains $1 \mu \mathrm{g}$ of pDNA. Lane 1, DNA molecular weight marker; lane 2, free pDNA; lane 3, free pDNA incubated with rhDNase-I; lane 4, free pDNA incubated with inhibited DNaseI; lane 5, microbubbles and pDNA; lane 6, microbubbles and pDNA incubated with DNase-I; lane 7, microbubbles and pDNA incubated with inhibited DNase-I. Smiling appeared because of the high salt concentrations used $(5 \mathrm{M} \mathrm{NaCl})$.

Figure 7 shows the gel electrophoresis results on pDNA released from pDNA-loaded microbubbles (by $\mathrm{NaCl}$ ) that were exposed to DNase-I. As shown in Figure 7A, DNase-I degrades both free pDNA (lane 3) and pDNA in the presence of uncoated microbubbles (lane 6). In contrast, the pDNA on PAH-coated microbubbles remains mainly protected (Figure 7B) because only a very small part of degraded pDNA seems to be present (arrow in lane 6). The latter can be explained by the fact that in this experiment a small amount of the pDNA remained in the solution as the maximal loading capacity of the microbubbles was exceeded. Clearly, in Figure 7B (lanes 5-7) a part of the pDNA remained in the slots of the agarose gel. Most presumably, this is attributed to the fact that, besides free pDNA, (high molecular weight) pDNA-PAH complexes were also liberated from the microbubbles upon adding $\mathrm{NaCl}$. This implies that the electrostatic binding between DNA and PAH is stronger than the interaction between the albumin and PAH. Thus, a second advantage of the PAH-coated microbubbles compared to the microbubbles of Frenkel's group is that they also protect DNA against DNase-I degradation.

From Figure 7B (lanes 2 and 5), we can also conclude that no conformational change of the pDNA occurred upon binding

(24) De Geest, B. G.; Dejugnat, C.; Sukhorukov, G. B.; Braeckmans, K.; De Smedt, S. C.; Demeester, J. Adv. Mater. 2005, 17, 2357.

(25) Park, M. K.; Deng, S. X.; Advincula, R. C. Langmuir 2005, 21, 5272

(26) Zahr, A. S.; de Villiers, M.; Pishko, M. V. Langmuir 2005, 21, 403.

(27) Kabalnov, A.; Bradley, J.; Flaim, S.; Klein, D.; Pelura, T.; Peters, B.; Otto, S.; Reynolds, J.; Schutt, E.; Weers, J. Ultrasound Med. Biol. 1998, $24,751$. (28) Riess, J. G. Curr. Opin. Colloid Sci. 2003, 8, 259.

(29) Shchukin, D. G.; Kohler, K.; Mohvald, H.; Sukhorukov, G. B. Angew. Chem., Int. Ed. 2005, 44, 3310 .

(30) Stein, C. A.; Cheng, Y. C. Science 1993, 261, 1004.

(31) Frenkel, P. A.; Chen, S. Y.; Thai, T.; Shohet, R. V.; Grayburn, P. A. Ultrasound Med. Biol. 2002, 28, 817.

(32) Kawai, Y.; Yoshida, M.; Arakawa, K.; Kumamoto, T.; Morikawa, N.; Masamura, K.; Tada, H.; Ito, S.; Hoshizaki, H.; Oshima, S.; Taniguchi, K.; Terasawa, H.; Miyamori, I.; Kishi, K.; Yasuda, T. Circulation 2004, 109, 2398. 
to the microbubbles. Indeed, the released DNA (lane 5) is visible at the same height on the gel as the free pDNA (lane 2).

\section{Conclusions}

A major issue in ultrasound-assisted gene transfer is the development of microbubbles that both bind and protect pDNA against circulating DNases in the bloodstream. It is well known that binding pDNA with cationic polymers or lipids protects the pDNA against degradation by DNase I. ${ }^{1}$ Therefore, we evaluated the possibility of coating the medically used albumin/perfluorocarbon contrast agents with a cationic polymer. A successful coating of the albumin-shelled perfluorocarbon microbubbles with PAH was evidenced from CLSM and zeta potential measurements. The positive charges on the surface of the PAHcoated microbubbles allowed the binding of up to $0.1 \mathrm{pg}$ of pDNA on the wall of a single microbubble. The pDNA bound on PAH-coated microbubbles was clearly protected against nucleases. The presence of pDNA on the microbubble may also enhance the number of pDNA molecules that can enter a cell during sonoporation. Indeed, small pores (up to $100 \mathrm{~nm}$ large) created by imploding microbubbles have a very short lifetime (millisecond range). ${ }^{16}$ To increase the number of pDNA molecules that pass through these quickly closing pores, it seems clear that it is important to co-localize DNA and pore-forming microbubbles. Furthermore, PAH coating of the microbubbles improved their stability, thereby increasing their (average) lifetime from $75 \mathrm{~min}$ to $6 \mathrm{~h}$ while they remained ultrasound-responsive.
Their longer lifetime makes them more convenient for in vivo therapy because more microbubbles are expected to reach the target organ. In this study, we used PAH as a cationic polymer to coat the microbubbles. This polymer is not suitable for in vivo applications because, as a result of its high molecular weight and nonbiodegradability, it will not be efficiently cleared from the human body. However, it is obvious that other cationic polymers that are more biocompatible and biodegradable may also be used as coating material. Also, coating microbubbles with cationic polymers may be an attractive strategy to promote the targeting of microbubbles because it is rather straightforward to attach targeting ligands to the polymer coating. This could further enhance the site-specific delivery of pDNA and could provide an even higher pDNA concentration close to cell membrane perforations. ${ }^{33}$ However, to obtain good gene transfer it is also important that the pDNA dissociates from the microbubble upon ultrasound exposure. In our future work, we will tackle this issue and perform transfection experiments with our microbubbles.

Acknowledgment. I.L. is a doctoral fellow of FWO-Flanders. N.N.S. is a postdoctoral fellow of FWO (Fund for Scientific Research-Flanders). The financial support of this institute is acknowledged with gratitude. We thank Professor Vervaet and Evy Corbany for the use of the laser diffractor. We thank Olivier Janssens and Philippe Smet for taking the SEM images.

LA0603828

(33) Klibanov, A. L. Bioconjugate Chem. 2005, 16, 9. 